\author{
UNIVERSIDADE DE SÃO PAULO \\ FACULDADE DE ODONTOLOGIA DE BAURU
}

CARLA RUFFEIL MOREIRA

Validaçáo de medidas maxilofaciais por meio da tomografia computadorizada por feixe cônico em 3D 

CARLA RUFFEIL MOREIRA

Validação de medidas maxilofaciais por meio da tomografia computadorizada por feixe cônico em 3D

Tese apresentada à Faculdade de Odontologia de Bauru da Universidade de São Paulo para obtenção do título de doutor em Odontologia.

Área de Concentração: Estomatologia Orientador: Prof. Dr. Marcelo de Gusmão Paraíso Cavalcanti 


\begin{tabular}{|} 
Moreira, Carla Ruffeil \\
M813v Validação de medidas maxilofaciais por meio da \\
tomografia computadorizada por feixe cônico em 3D / \\
Carla Ruffeil Moreira. - Bauru, 2009. \\
75 p. : il. ; $30 \mathrm{~cm}$. \\
Tese. (Doutorado) - Faculdade de Odontologia de \\
Bauru. Universidade de São Paulo. \\
Orientador: Prof. Dr. Marcelo de Gusmão Paraíso \\
Cavalcanti
\end{tabular}

Autorizo, exclusivamente para fins acadêmicos e científicos, a reprodução total ou parcial desta tese, por processos fotocopiadores e outros meios eletrônicos.

Assinatura:

Data:

Comitê de Ética da FO-USP Protocolo no $133 / 07$

Data: 04 de outubro de 2007 
FOLHA DE APROVAÇÃO 



\section{DADOS CURRICULARES}

10 de janeiro de 1979

Filiação

$1997-2002$

$1998-2001$

2003-2005

2006-2007

2005-2009
Nascimento

Belém - PA

Carlos Guilherme Lavor Moreira

Maria da Conceição Ruffeil Moreira

Curso de Odontologia - Faculdade de Odontologia, Universidade Federal do Pará

Programa de Iniciação Científica - PIPES - UFPA

Curso de Pós-Graduação em nível de Mestrado Faculdade de Odontologia de Bauru - USP

Especialização em Radiologia Odontológica e Imaginologia - Associação Paulista de Cirurgiões Dentistas - APCD - Bauru - SP

Curso de Pós-Graduação em nível de Doutorado Faculdade de Odontologia de Bauru - USP 

Aos meus pais Carlos e Conceição, ao meu irmão Marcos e à minha família 



\section{Agradecimentos especiais}

Ao Prof. Dr. José Humberto Damante, que me acolheu durante toda a pósgraduação, acreditando no meu trabalho e proporcionando muito da minha formação.

Ao meu orientador, Prof. Dr. Marcelo de Gusmão Paraíso Cavalcanti. Agradeço a valiosa contribuição não apenas para a realização deste estudo, mas para a minha produção científica durante o doutorado. Obrigada pela confiança, pelo apoio e principalmente pela amizade construída ao longo desses anos.

Ao amigo Prof. Dr. Marcelo Augusto Oliveira Sales, por ter participado ativamente do trabalho, sempre com muita dedicação e presteza. 



\section{Agradecimentos}

À Faculdade de Odontologia de Bauru, em nome do seu diretor, Prof. Dr. Luiz Fernando Pegoraro.

À Faculdade de Odontologia de São Paulo, especialmente ao Laboratório de Imagens em 3D (Labi-3D).

À Coordenação de Aperfeiçoamento de Pessoal de Nivel Superior - CAPES, pelo investimento através da bolsa de doutorado.

Aos professores do Departamento de Estomatologia da FOB, especialmente à Profa. Dra. Ana Lúcia Álvares Capelozza, à Profa. Dra. Izabel Regina Fischer Rubira-Bullen e ao Prof. Dr. Luiz Eduardo Montenegro Chinellato, pelos ensinamentos e pela amizade.

A todos os funcionários da Faculdade de Odontologia de Bauru, especialmente aos funcionários do Departamento de Estomatologia, Marîlia Gião, Fernanda Cavalari, Roberto Sales, Josieli Farinha, Elza Cassalate, Luciana Lozano e Alexandre Garcia por toda a solicitude.

Aos professores e funcionários da Faculdade de Odontologia de São Paulo - FOUSP, especialmente ao Prof. Dr. José Leopoldo Ferreira Antunes pela atenção e pelos conhecimentos transmitidos.

À Dra. Maura Ito da Alpha X Radiologia Odontológica (Barueri, SP), por permitir a realização das tomografias computadorizadas.

Ao Centro Universitārio de João Pessoa - UNIPE pela atualização do programa utilizado neste trabalho. 

Ao Prof. Dr. Ricardo Smith do Departamento de Morfologia da Escola Paulista de Medicina - UNIFESP, pela gentileza e concessão dos crânios.

Às professoras da Universidade Federal do Pará, Profa. Dra. Cecy Martins Silva e Profa. Dra. Regina Feio Barroso, pela orientação durante a iniciação científica.

Aos professores e funcionários da Universidade Federal do Pará, especialmente ao Prof. Armando Chermont, Prof. Armando Ferreira, Prof. Dr. Erick Pedreira, Prof. Dr. Fabrício Tuji, Profa. Dra. Flāvia Pontes, Prof. Dr. Hélder Pontes e Prof. Dr. João Pinheiro, pelo incentivo constante.

Aos professores e funcionários da Escola Superior da Amazônia - Esamaz e ao efetivo do Hospital de Aeronáutica de Belém - HABE, pela compreensão e pelo apoio.

Aos amigos da pós-graduação da FOB, Angêlica Hannas, Cássia Rubira, Etiene Munhoz, Letícia Nery, Camila Lopes Cardoso, Marta da Cunha Lima, Ana Cláudia de Araújo Pires, Moacyr Rodrigues, Marcelo Junior Zanda, Gustavo Lautenschlager, Josiane Sá, Melissa Araujo, Renata Teixeira, Elen Tolentino, Gabriel Bernini, Daniele Albuquerque, Manuela Rodriguez, Renato Yaedu, Fernando Oliveira e Priscila Brenner, pelo companheirismo e pela amizade.

Aos integrantes do Labi-3D da FOUSP, Adriana Paes, Alexandre Marques, Caio Cremonini, Marianna Dumas, Estevam Utumi, Marco Albuquerque, Patrícia Lopes, Denise Takehana, Maurício Accorsi e, especialmente, à amiga Andreia Perrella, por tornarem o trabalho muito mais agradāvel.

A todos os amigos que, de alguma forma, contribuíram para a realização deste trabalho. 



\section{RESUMO}

O objetivo deste estudo foi demonstrar a precisão e a acurácia de medidas maxilofaciais lineares e angulares obtidas por tomografia computadorizada por feixe cônico (TCFC). A amostra consistiu de quinze crânios humanos secos submetidos à TCFC. Medidas lineares e angulares foram realizadas em imagens em terceira dimensão (3D) após a identificação de pontos craniométricos convencionais. As imagens em 3D-TCFC foram analisadas por dois radiologistas, duas vezes, independentemente. Medidas físicas foram realizadas por um terceiro examinador utilizando paquímetro e goniômetro digitais. Os resultados não demonstraram diferenças estatísticas significantes para as análises intra e interexaminadores. As comparações entre as medidas físicas e as obtidas em 3D-TCFC para ambos os examinadores também não foram estatisticamente significantes tanto para as medidas lineares quanto paras as angulares $(p=0,968$ e 0,915, $p=0,844$ e 0,700, respectivamente). As imagens em 3D-TCFC podem ser utilizadas com precisão e acurácia para a obtenção de medidas lineares e angulares a partir de estruturas anatômicas e pontos craniométricos.

Palavras-chave: Craniometria. Tomografia computadorizada de feixe cônico. Imagem Tridimensional. 



\section{ABSTRACT \\ Assessment of linear and angular measurements on three-dimensional cone- beam computed tomographic images}

The purpose of this research was to provide further evidence to demonstrate the precision and accuracy of maxillofacial linear and angular measurements obtained by cone-beam computed tomography (CBCT) images. The study population consisted of 15 dry human skulls that were submitted to a CBCT, and threedimensional (3D) images were generated. Linear and angular measurements based upon conventional craniometric anatomical landmarks, were identified in 3D-CBCT images by two radiologists twice each independently. Subsequently physical measurements were made by a third examiner using a digital caliper and a digital goniometer. The results demonstrated no statistically significant difference between inter and intra-examiner analysis. Regarding accuracy test, no statistically significant difference were found of the comparison between the physical and CBCT-based linear and angular for both examiners $(p=0.968$ and $0.915, p=0.844$ and 0.700 respectively). 3D-CBCT images can be used to obtain dimensionally accurate linear and angular measurements from bony maxillofacial structures and landmarks.

Keywords: Cephalometry. Cone-beam computed tomography. Imaging, ThreeDimensional. 



\section{SUMÁRIO}

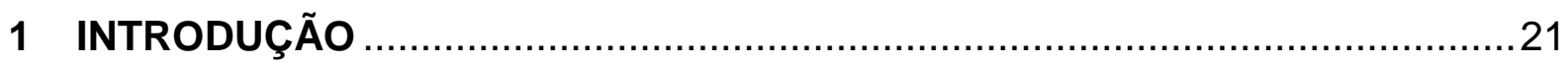

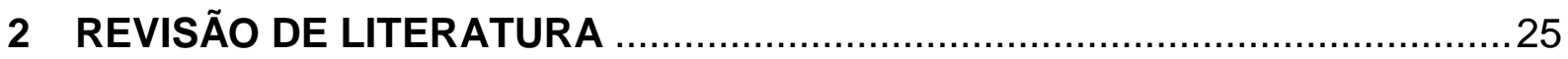

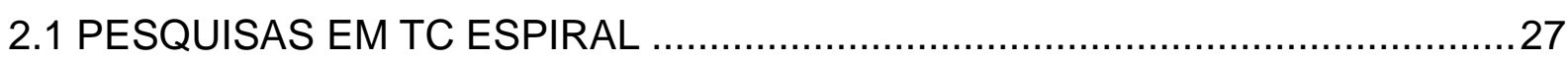

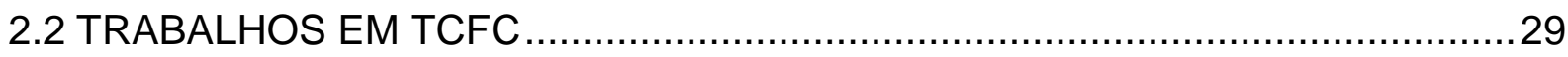

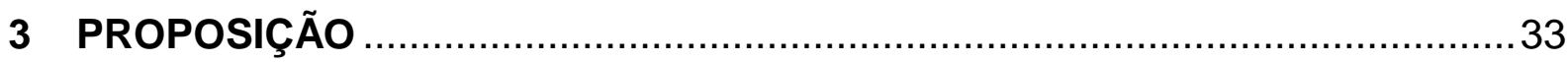

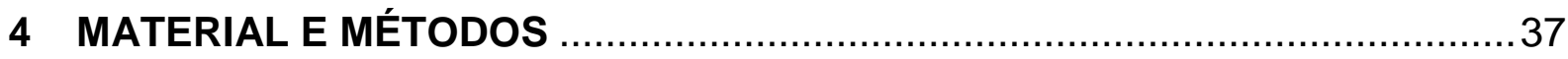

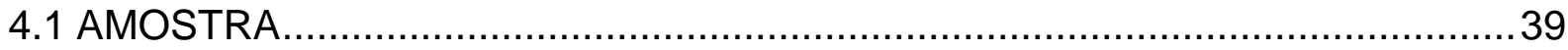

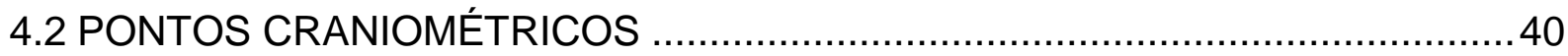

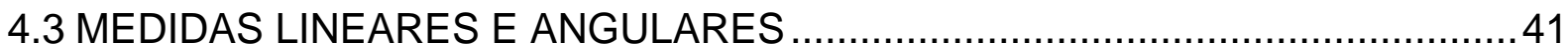

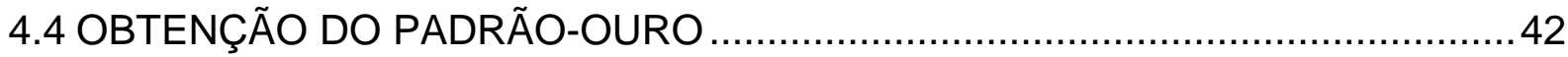

4.5 TOMOGRAFIA COMPUTADORIZADA POR FEIXE CÔNICO............................43

4.6 PROCESSAMENTO E RECONSTRUÇÃO DAS IMAGENS …............................44

4.7 OBTENÇÃO DAS MEDIDAS EM 3D-TCFC NO PROGRAMA VITREA ${ }^{\circledR} \ldots \ldots \ldots . . . .45$

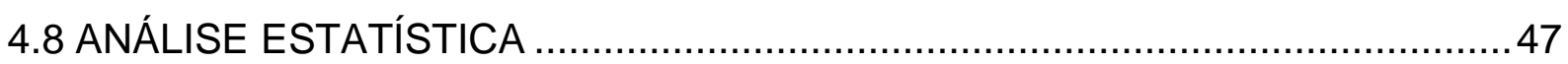

5 RESULTADOS

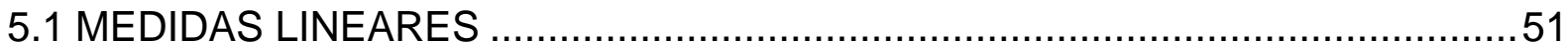

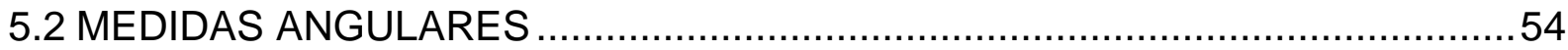

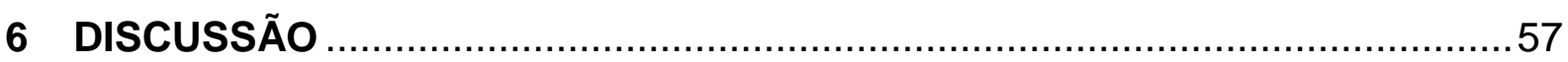

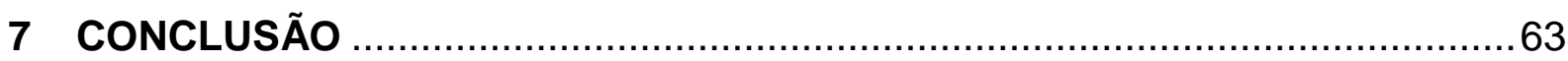

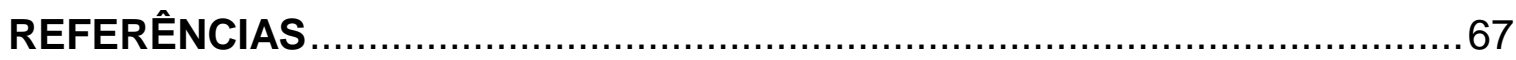

ANEXO 

1 INTRODUÇÃO 



\section{INTRODUÇÃO}

Os avanços da tomografia computadorizada (TC) a consolidaram como uma importante modalidade de imagem dentro da Odontologia. As aplicações da TC single e multislice na região craniofacial para diversos fins, como medidas craniométricas, deformidades craniofaciais, identificação forense e para o diagnóstico e planejamento cirúrgico de fraturas, implantes e lesões maxilofaciais estão bem descritas na literatura (JAMILSON e WARD, 1993; KRAGSKOV et al., 1997; CAVALCANTI e VANNIER, 1998; CAVALCANTI, RUPRECHT e VANNIER, 2002; CAVALCANTI, ROCHA e VANNIER, 2004; NAITOH et al., 2004).

Recentemente, a tomografia computadorizada por feixe cônico (TCFC), inicialmente descrita por Mozzo et al. (1998), tem sido largamente indicada para a região maxilofacial (SUKOVIC, 2003; FARMAN e SCARFE, 2006; SCARFE, FARMAN e SUKOVIC, 2006). A TCFC rapidamente ganhou espaço na Odontologia, principalmente por suas características de ausência de sobreposição de imagens, doses de radiação mais baixas, menor custo do aparelho e a possibilidade de fazer medidas a partir de reconstruções geradas por programas de computação gráfica (SUKOVIC, 2003; FARMAN e SCARFE, 2006; SCARFE, FARMAN e SUKOVIC, 2006).

Medidas lineares em TCFC têm sido estudadas e análises craniométricas propostas, porém as estruturas anatômicas e os critérios para o plano de tratamento precisam ser definidos (PINSKY et al., 2006; SWENNEN e SCHUTYSER, 2006; MOSHIRI et al. 2007; LAGRAVÈRE et al. 2008; PERIAGO et al.; 2008; STRATEMANN et al. 2008). Nesse contexto, a TC em espiral foi amplamente utilizada, o que resultou na validação de muitos experimentos (CAVALCANTI, ROCHA e VANNIER, 2004; LOPES, 2006; ACCORSI, 2007; LOPES et al., 2007, 2008). Porém, estudos comprovando a precisão e a acurácia da TCFC são escassos e maiores esclarecimentos ainda são necessários. 

2 REVISÃO DE LITERATURA 



\section{REVISÃO DE LITERATURA}

\subsection{PESQUISAS EM TC ESPIRAL}

A precisão e a acurácia das medidas craniométricas nas imagens de tomografia computadorizada espiral em terceira dimensão (3D) já foi realizada por vários autores (CAVALCANTI e VANNIER, 1998; CAVALCANTI, RUPRECHT e VANNIER, 2002; CAVALCANTI, ROCHA e VANNIER, 2004; LOPES et al., 2007, 2008).

Cavalcanti e Vannier (1998) avaliaram a acurácia das mensurações craniofaciais obtidas por meio da TC espiral nas reconstruções em 2D e 3D. Medidas foram feitas a partir de pontos craniométricos identificados nas imagens em 2D-TC e em 3D-TC em nove cabeças de cadáveres por 02 examinadores, duas vezes cada. O tecido mole foi removido dos cadáveres e as mensurações realizadas por um digitalizador eletromagnético. Os resultados mostraram que todas as mensurações em 3D-TC tiveram uma acurácia satisfatória em comparação com as medidas físicas.

Cavalcanti, Ruprecht e Vannier (2002) validaram a TC em espiral para o planejamento de implante dentário. Oito cabeças de cadáveres foram avaliadas por dois examinadores, que mediram da borda superior do forame mentual até a crista do processo alveolar e da borda inferior do forame mentual até a borda inferior da mandíbula, bilateralmente. As medidas físicas foram realizadas com o 3 space $^{T M}$ digitalizer eletromagnético, após dissecção da área de interesse. O programa CEMAX foi utilizado para obtenção das imagens volumétricas em 2D-TC e o protocolo Tooth Pix ${ }^{T M}$ para manipulação e análise. Para os casos de implantes com proximidade do forame mentual, a TC em espiral permitiu um alto grau de acurácia para o planejamento cirúrgico. Os autores concluíram que os programas em 3D-TC permitiram melhor visualização da anatomia facial em relação às estruturas adjacentes.

Cavalcanti, Rocha e Vannier, em 2004, estudaram a aplicabilidade da computação gráfica em 3D-TC com o propósito de estabelecer um método de 
reconstrução facial. Quinze cabeças de cadáveres foram submetidas à TC espiral e avaliadas no programa $V_{i t r e a}{ }^{\circledR}$. Dez medidas craniométricas lineares foram realizadas por dois observadores, independentemente, 2 vezes cada. As medidas físicas foram obtidas por um terceiro observador através do 3D digitizer eletromagnético. Os resultados demonstraram precisão e acurácia das medidas lineares determinadas na 3D-TC, tanto nas tegumentares quanto nas ósseas. Os pesquisadores concluíram que a computação gráfica ofereceu recursos valiosos para tornar a análise craniométrica mais fácil e rápida para a Odontologia Forense.

Em 2006, Park et al. propuseram uma nova análise craniofacial através da 3D-TC. Imagens tridimensionais de 30 indivíduos coreanos submetidos à TC foram geradas e medidas nos programas Vworks 4.0 e Vsurgery, respectivamente. Pontos craniométricos $(n=19)$ foram identificados para medidas lineares e angulares. Os dados obtidos foram confrontados com as médias da população coreana e não foram observadas diferenças estatisticamente significantes. Os autores concluíram que a análise 3D pode fornecer informações úteis no diagnóstico e plano de tratamento.

Lopes et al., em 2007, avaliaram a precisão e a acurácia de medidas cefalométricas lineares em imagens em 3D. Dez crânios secos foram submetidos à TC multislice 16 canais e analisados no programa Vitrea ${ }^{\circledR}$. Pontos cefalométricos $(n=13)$ foram localizados e medidas lineares $(n=15)$ realizadas por 2 examinadores, duas vezes cada. As medidas físicas foram obtidas por um terceiro examinador, utilizando um paquímetro digital. Não houve diferenças estatisticamente significantes nas análises intra e interexaminadores, nem entre as medidas físicas e em 3D. O erro percentual para as medidas inter e intra-examinadores foi de 2,05\% e 2,11\%, respectivamente. A média do erro percentual entre as medidas físicas e em 3D variou de $0,96 \%$ a $1,47 \%$. Assim, todas as medidas cefalométricas lineares foram consideradas precisas e acuradas na 3D-TC multislice.

Seguindo a mesma linha de pesquisa, Lopes et al., em 2008, estudaram a precisão e a acurácia de medidas cefalométricas angulares por meio de reconstruções em 3D, utilizando a TC multislice 64 canais. Vinte e oito crânios secos foram submetidos à TC e analisados no programa Vitrea ${ }^{\circledR}$. Pontos cefalométricos ( $n=$ 9) foram localizados nas imagens em 3D-TC e medidas angulares $(n=6)$ realizadas por dois examinadores, duas vezes cada. Medidas físicas foram obtidas por um 
terceiro examinador através do aparelho Beyond Crysta-C 9168. Os resultados não demonstraram diferenças estatisticamente significantes entre as medidas em 3D-TC e as medidas físicas e nem nas medidas inter e intra-examinadores.

\subsection{TRABALHOS EM TCFC}

Pinsky et al. (2006) realizaram um estudo in vitro em defeitos intraósseos avaliando a acurácia de medidas lineares e volumétricas em TCFC. Simularam defeitos ósseos pequenos parecidos com aqueles causados por lesões periodontais e periapicais. Ressaltaram a dificuldade de diagnóstico dessas lesões usando radiografias convencionais pela bidimensionalidade e a aplicabilidade da TCFC em Odontologia. Sessenta e quatro lesões foram confeccionadas em um bloco de acrílico e vinte e uma em uma mandíbula humana e medidas através de um programa de computação por cinco avaliadores, duas vezes cada, com intervalo de 7 dias entre elas. Os autores consideraram a utilização da TCFC prática, acurada e não invasiva para determinar o tamanho e o volume dos defeitos.

Kumar et al., em 2007, confrontaram os resultados de medidas lineares e angulares em radiografias cefalométricas com aquelas obtidas em projeções 2D oriundas de TCFC. Nove medidas lineares e cinco angulares foram realizadas em dez crânios humanos secos por três vezes. A projeção 2D em TCFC forneceu maior acurácia nas mensurações quando comparada à radiografia. Apesar de esse estudo englobar medidas lineares e angulares, estas não foram realizadas em terceira dimensão.

Também em 2007, Moshiri et al. compararam a acurácia de medidas lineares obtidas em radiografias digitais e em TCFC. Quinze pontos craniométricos foram marcados em vinte e três crânios humanos secos por dois observadores, três vezes cada. As medidas foram realizadas em projeções em 2D oriundas de TCFC simulando radiografias cefalométricas laterais. As imagens em TCFC mostraram maior acurácia que as radiografias digitais. Foi observada uma tendência de magnificação variando de 4,6 a 9\% nas radiografias digitais. 
Periago et al. (2008) testaram a acurácia de medidas lineares realizadas em 3D-TCFC. Pontos craniométricos $(n=14)$ e medidas lineares $(n=20)$ comumente usados em Ortodontia foram identificados e obtidos em 23 crânios humanos secos através de um paquímetro digital (padrão-ouro) e virtualmente no programa Dolphin $3 D$ versão 2.3. A realização de medidas lineares em imagens $3 D$ foi tida como acurada para análise craniofacial. Apesar de terem encontrado diferenças estatisticamente significantes em algumas medidas, os autores não as consideraram clinicamente importantes.

Em 2008, Lagravère et al. avaliaram a acurácia de medidas lineares e angulares realizadas em TCFC. Uma mandíbula sintética com dez marcadores de titânio fixados foi avaliada por um examinador. As medições foram realizadas em imagens em 3D, que proporcionaram mensurações em tamanho real e não houve diferenças estatisticamente significantes entre a TCFC e o padrão-ouro. Porém, não foi realizada a marcação de pontos craniométricos, já que as distâncias obtidas foram entre os marcadores metálicos. Os autores ressaltaram a acurácia das imagens em 3D e a necessidade do estabelecimento de parâmetros para serem seguidos na prática clínica, especialmente voltada para as intervenções ortodônticas.

Yamashina et al., ainda em 2008, compararam a confiabilidade da TCFC com a TC multislice em mensurações da região da orofaringe. Um fantom e um voluntário foram submetidos às duas tomografias para medição do espaço aéreo. Apesar das diferenças observadas nos valores das unidades Hounsfield, tanto a TC quanto a TCFC foram consideradas acuradas para a mensuração do volume do espaço aéreo circundado por tecidos moles.

Stratemann et al., também em 2008, realizaram um estudo para determinar a acurácia de medidas lineares usadas em Ortodontia através de dois aparelhos de TCFC. Esferas metálicas foram encravadas em pontos craniométricos de um crânio humano seco. As medidas lineares foram obtidas através de um paquímetro digital e confrontadas com as realizadas nos dois tipos de TCFC. Os resultados mostraram acurácia alta para ambos os sistemas de TCFC, com menos de $1 \%$ de erro.

Oliveira et al., em 2009, testaram a confiabilidade na identificação de pontos craniométricos em imagens sagitais, coronais e axiais de TCFC. Três observadores marcaram 30 pontos em doze exames de TCFC, separadamente, três vezes cada, 
com intervalos de pelo menos três dias entre as avaliações. Os resultados intra e interexaminadores foram considerados excelentes. Apesar das vantagens da cefalometria em 3D terem sido citadas, os autores atribuíram o uso restrito dessas imagens na prática clínica ao maior tempo gasto em análises desta natureza e à necessidade de familiaridade com a informática.

A partir dessa revisão, nota-se na literatura a escassez de trabalhos em 3DTCFC e, ainda, que reproduzam condições clínicas de identificação de pontos craniométricos e realização de medidas maxilofacias lineares e angulares. 

3 PROPOSICÃO 



\section{PROPOSIÇÃO}

Avaliar a precisão e a acurácia de medidas maxilofaciais lineares e angulares obtidas por TCFC. Para tanto, propomo-nos a:

- Confrontar as medidas maxilofaciais lineares e angulares obtidas a partir de reconstruções tridimensionais de TCFC com aquelas consideradas padrão-ouro;

- Verificar a precisão intra-examinador e interexaminador na realização dessas medidas maxilofaciais.

A partir desses dados, objetivamos determinar a aplicabilidade da TCFC na execução de medidas craniométricas em imagens tridimensionais. 

4 MATERIAL E MÉTODOS 



\section{MATERIAL E MÉTODOS}

\subsection{AMOSTRA}

A amostra foi constituída por 15 (quinze) crânios secos (12 homens e 3 mulheres) com idades variando entre 19 e 56 anos, previamente selecionados, sem distinção de etnia ou gênero, pertencentes ao Departamento de Morfologia da Escola Paulista de Medicina da Universidade Federal de São Paulo (EPM UNIFESP). O projeto teve aprovação do comitê de ética da Faculdade de Odontologia de São Paulo (FOUSP). 


\subsection{PONTOS CRANIOMÉTRICOS}

Os catorze pontos craniométricos adotados foram pontos convencionais previamente utilizados por Cavalcanti e Vannier (1998), Cavalcanti, Rocha e Vannier (2004) e Lopes et al. (2007, 2008). (Tabela 1)

Tabela 1 - Pontos craniométricos

\begin{tabular}{|c|c|}
\hline Ponto & Localização \\
\hline Pório (Po) & $\begin{array}{l}\text { Ponto mais alto da margem superior do meato } \\
\text { auditivo externo }\end{array}$ \\
\hline Básio (Ba) & $\begin{array}{l}\text { Ponto localizado no limite ínfero-posterior da borda } \\
\text { anterior do forame magno }\end{array}$ \\
\hline Condílio (Co) & $\begin{array}{l}\text { Ponto mais posterior e superior da cabeça da } \\
\text { mandíbula }\end{array}$ \\
\hline Espinha nasal anterior (ENA) & Ponto mais anterior do assoalho das fossas nasais \\
\hline Espinha nasal posterior (ENP) & Ponto mais posterior do assoalho das fossas nasais \\
\hline Násio (N) & $\begin{array}{l}\text { Ponto localizado na porção mais anterior da sutura } \\
\text { frontonasal }\end{array}$ \\
\hline Orbitário (Or) & Ponto mais inferior do assoalho da órbita \\
\hline A & $\begin{array}{l}\text { Ponto mais profundo da concavidade alveolar da } \\
\text { maxila }\end{array}$ \\
\hline B & $\begin{array}{l}\text { Ponto mais profundo da concavidade alveolar da } \\
\text { mandíbula }\end{array}$ \\
\hline Pogônio (Pg) & Ponto mais anterior da sínfise mentoniana \\
\hline Mentoniano (Me) & Ponto mais inferior e anterior da sínfise mentoniana \\
\hline Gônio (Go) & $\begin{array}{l}\text { Ponto mais posterior e inferior da curva entre } \\
\text { corpo e o ramo da mandíbula }\end{array}$ \\
\hline Zigomaxilar (Zm) & $\begin{array}{l}\text { Ponto mais inferior na sutura entre os ossos } \\
\text { zigomático e maxilar }\end{array}$ \\
\hline Glabela (G) & Ponto mais anterior do crânio no plano sagital \\
\hline
\end{tabular}




\subsection{MEDIDAS LINEARES E ANGULARES}

Quinze medidas lineares em milímetros e seis medidas angulares em graus (Tabela 2) foram realizadas por 02 observadores, medindo duas vezes cada um, independentemente, com intervalo de uma semana entre as duas avaliações.

Tabela 2 - Medidas lineares e angulares

\section{Medidas}

\begin{tabular}{|c|c|c|}
\hline & Lineares & Angulares \\
\hline 1 & $\mathrm{~A}-\mathrm{Pg}$ & N.A.Pg \\
\hline 2 & Co-A & G.ENA.Pg \\
\hline 3 & Co-Pg & Co.Go.Me \\
\hline 4 & ENA-ENP & N.Me.Go \\
\hline 5 & ENA-Me & N.A.B \\
\hline 6 & ENA-N & N.ENA.Me \\
\hline 7 & ENA-A & \\
\hline 8 & $N-A$ & \\
\hline 9 & $N-B$ & \\
\hline 10 & $\mathrm{~N}-\mathrm{Me}$ & \\
\hline 11 & $\mathrm{~N}-\mathrm{Pg}$ & \\
\hline 12 & Po-Or & \\
\hline 13 & $\mathrm{Zm}-\mathrm{Zm}$ & \\
\hline 14 & B-Me & \\
\hline 15 & $\mathrm{Ba}-\mathrm{N}$ & \\
\hline
\end{tabular}




\subsection{OBTENÇÃO DO PADRÃO-OURO}

O padrão-ouro do estudo foram as medidas físicas obtidas diretamente nos crânios secos, uma única vez, por um terceiro examinador que não teve conhecimento das medidas nas imagens em 3D. As medidas físicas lineares foram realizadas nas dependências do Departamento de Morfologia da EPM - UNIFESP utilizando-se um paquímetro digital, (Série 167, Mitutoyo Sul Americana Ltda, Suzano, SP, Brasil) com 0,3 mm de espessura de ponta. Este instrumento de medição foi desenvolvido pela Mitutoyo especialmente para pesquisas dessa natureza (LOPES, 2006; ACCORSI, 2007; LOPES et al., 2007). (Figura 1)
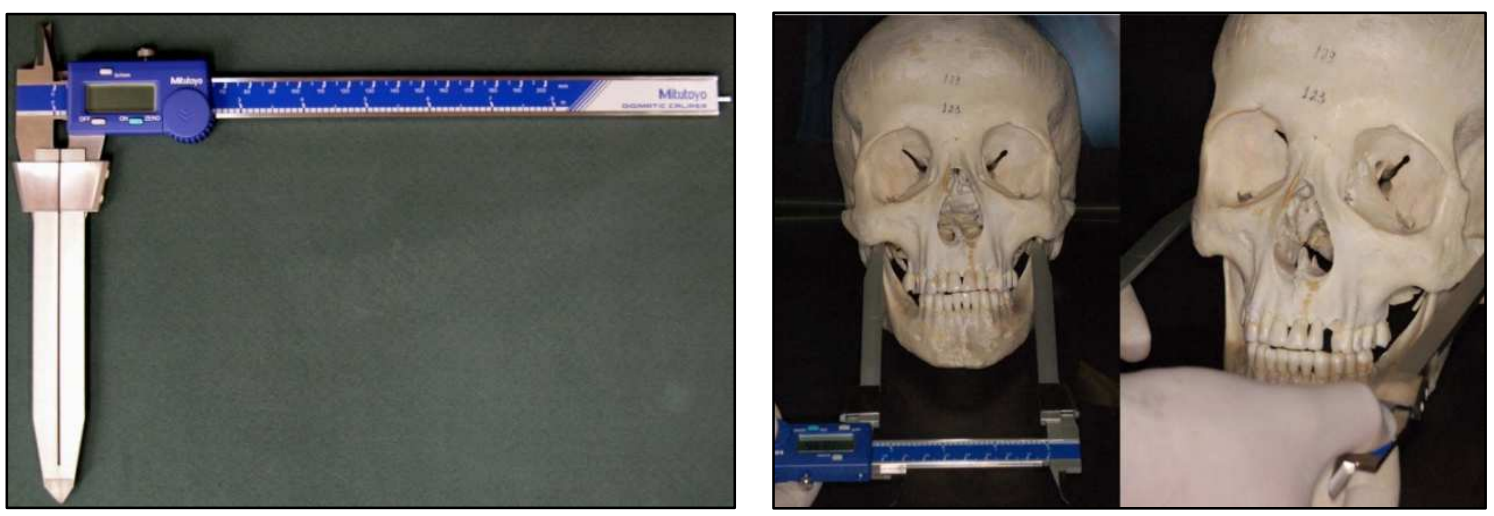

Figura 1 - Paquímetro digital

As medidas físicas angulares foram obtidas através de um goniômetro digital (Beyond Crysta-C 9168 series 900, Mitutoyo Sul Americana Ltda, Suzano, SP, Brasil) com ponta ativa de $0,3 \mathrm{~mm}$, localizado no Laboratório de Referência em Medição por Coordenadas (Mitutoyo Sul Americana Ltda, Suzano, SP, Brasil). As informações das mensurações foram coletadas em um programa específico (Geopak-Win Versão 2.4 R.8 Edição 10, Mitutoyo, Neuss, Alemanha) que calculou os dados e gerou as medidas angulares. Este aparelho teve a calibração certificada pelo Inmetro (Instituto Nacional de Metrologia, Brasil) e teve sua aplicabilidade para medidas maxilofaciais demonstrada anteriormente (LOPES et al., 2008). (Figura 2) 

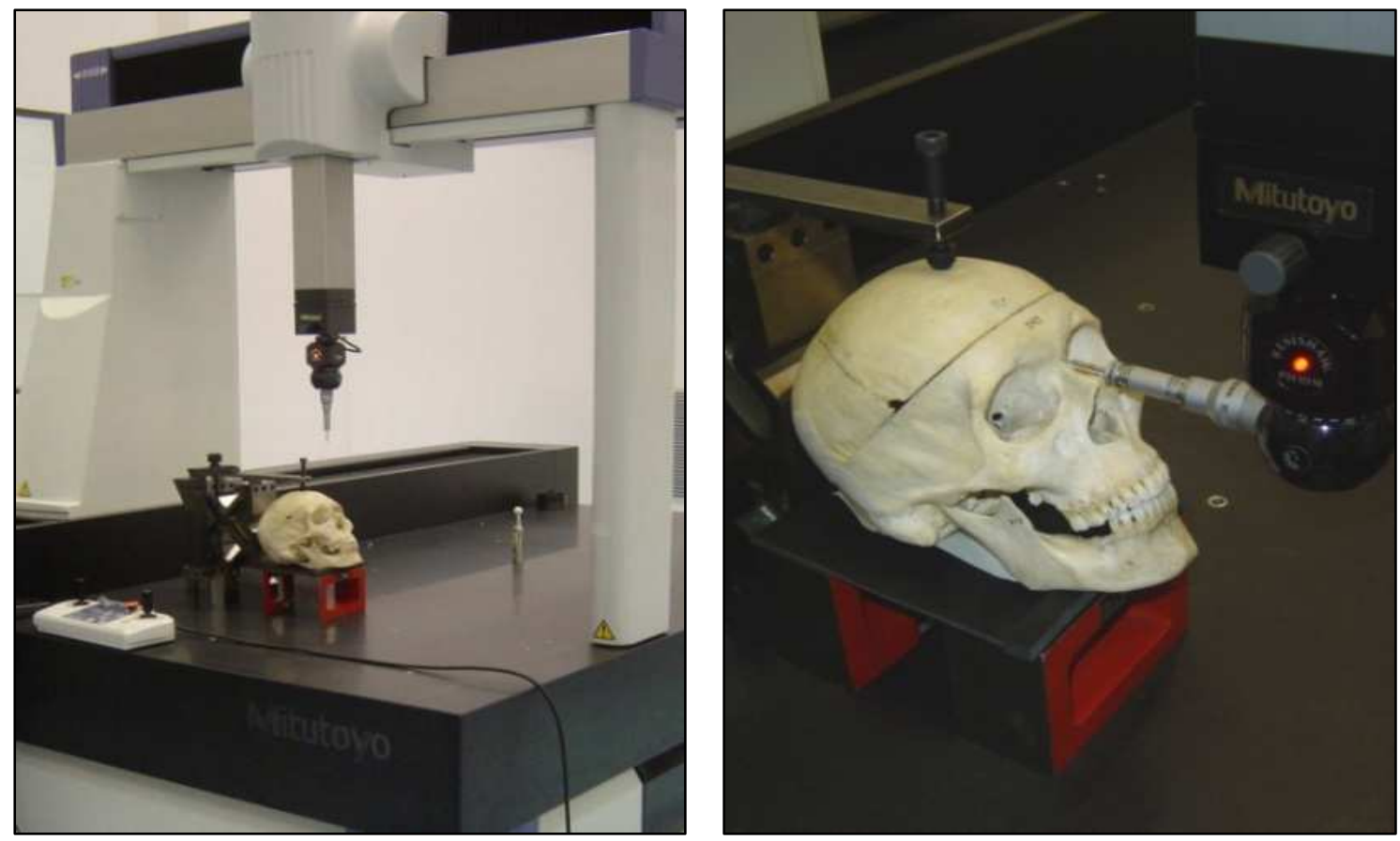

Figura 2 - Goniômetro digital

\subsection{TOMOGRAFIA COMPUTADORIZADA POR FEIXE CÔNICO}

Os espécimes foram submetidos à tomografia computadorizada por feixe cônico utilizando-se o aparelho i-CAT ${ }^{\circledR}$ Cone Beam 3-D Dental Imaging System (Imaging Sciences International, Hatfield, PA, EUA) com 0,25 mm de tamanho de voxel e 40 segundos de aquisição dos dados originais. As mandíbulas foram encaixadas nos crânios em máxima intercuspidação e fixadas através de fitas adesivas. Os crânios foram colocados dentro de um saco plástico grosso preenchido com água para simular a atenuação dos tecidos moles. Os crânios foram posicionados no equipamento usando o apoio do mento e o imobilizador da cabeça com o plano sagital mediano perpendicular ao solo e mantendo um posicionamento similar ao utilizado para exames em pacientes. (Figura 3) 


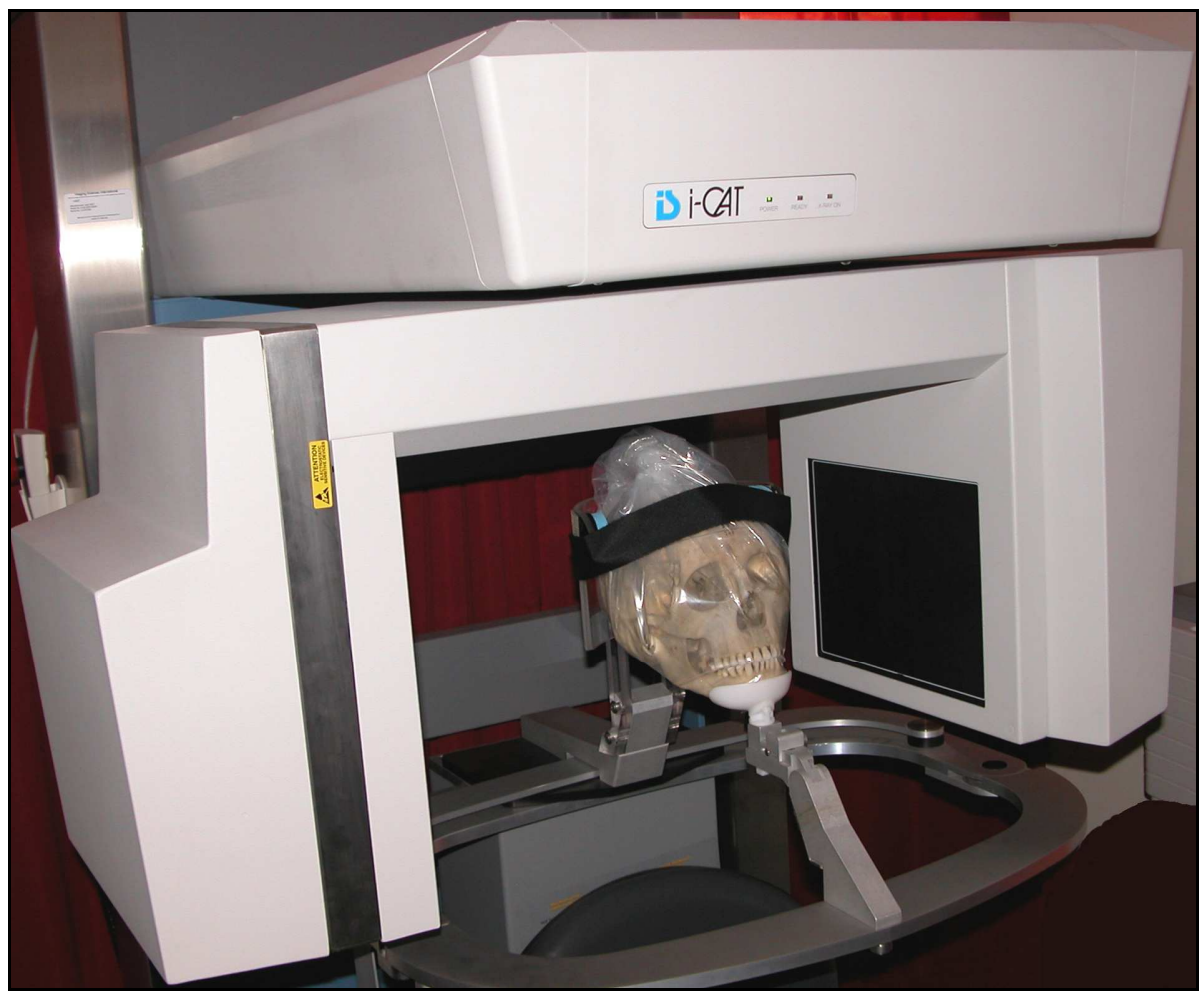

Figura 3 - Crânio posicionado no aparelho

\subsection{PROCESSAMENTO E RECONSTRUÇÃO DAS IMAGENS}

Os dados originais da TC foram armazenados em forma digital para possibilitar a revisão de qualquer imagem sem perda de qualidade, permitindo a geração de imagens volumétricas em 3D para processamento, visualização, manipulação e análise a qualquer momento. Posteriormente, estes dados foram transferidos para uma estação de trabalho independente (Dell Precision 650, Dell Computer Corp., Round Rock, TX, EUA) no formato DICOM (Digital Imaging and Communication in Medicine) localizada no Laboratório de Imagem em Terceira Dimensão (LABI-3D) da FOUSP. As imagens das reconstruções multiplanares e em 3D foram realizadas na estação de trabalho utilizando o programa Vitrea ${ }^{\circledR}$ versão 3.8.1.1 (Vital Images Inc., Plymouth, MN, EUA). 


\subsection{OBTENÇÃO DAS MEDIDAS EM 3D-TCFC NO PROGRAMA VITREA ${ }^{\circledR}$}

O programa Vitrea ${ }^{\circledR}$ permite a observação simultânea das imagens sagitais, coronais, axias e em 3D (Figura 4). Além disso, possibilita a rotação das imagens em 3D e a conseqüente análise em ângulos diversos usando a ferramenta transparência. Todos esses recursos foram amplamente utilizados na realização das medidas lineares e angulares (Figura 5). Para cada crânio, todos os pontos craniométricos foram localizados durante uma sessão de análise e as medidas resultantes foram obtidas utilizando a ferramenta régua do programa.

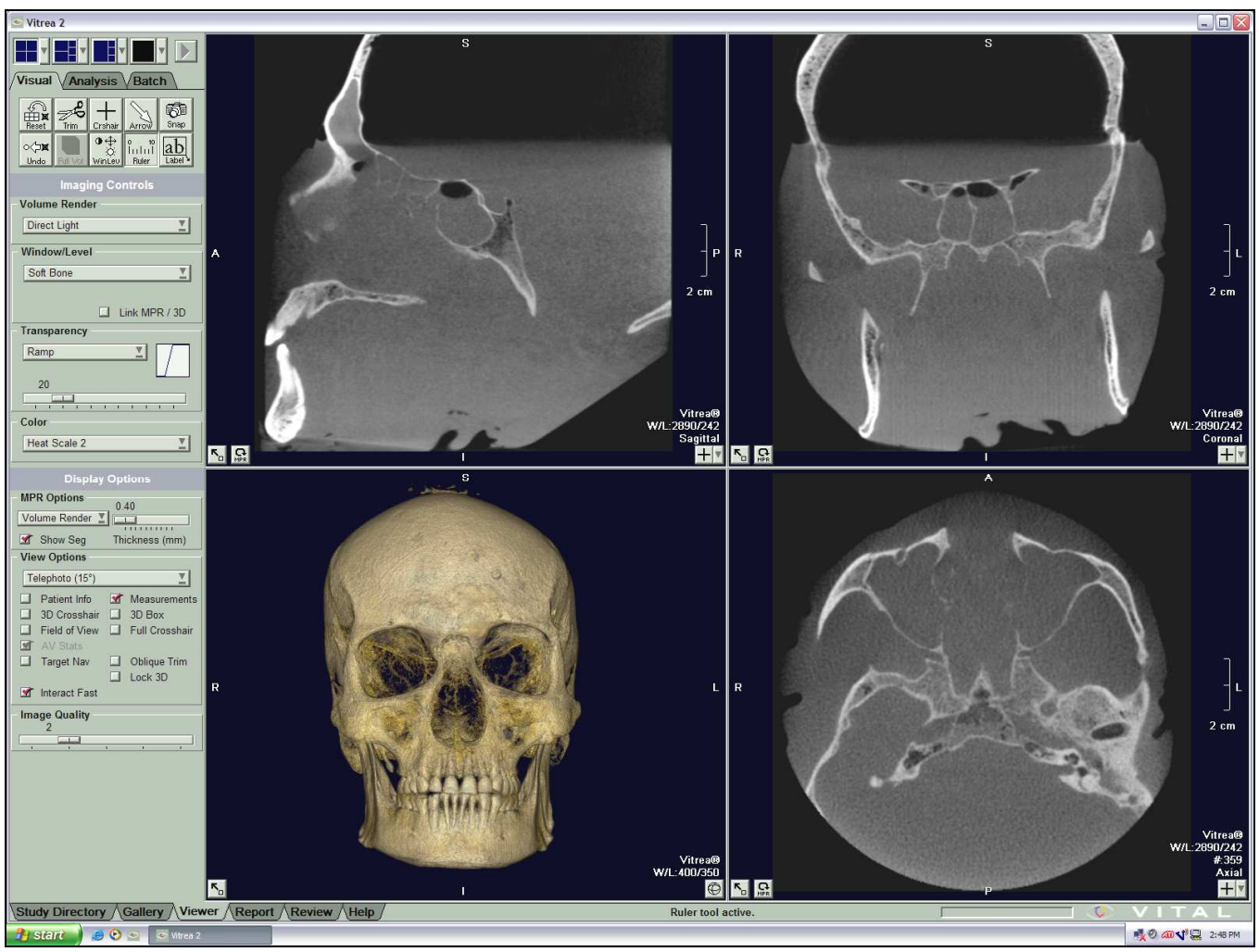

Figura 4 - Tela de abertura do programa mostrando a observação simultânea das imagens sagitais, coronais, axiais e em terceira dimensão 


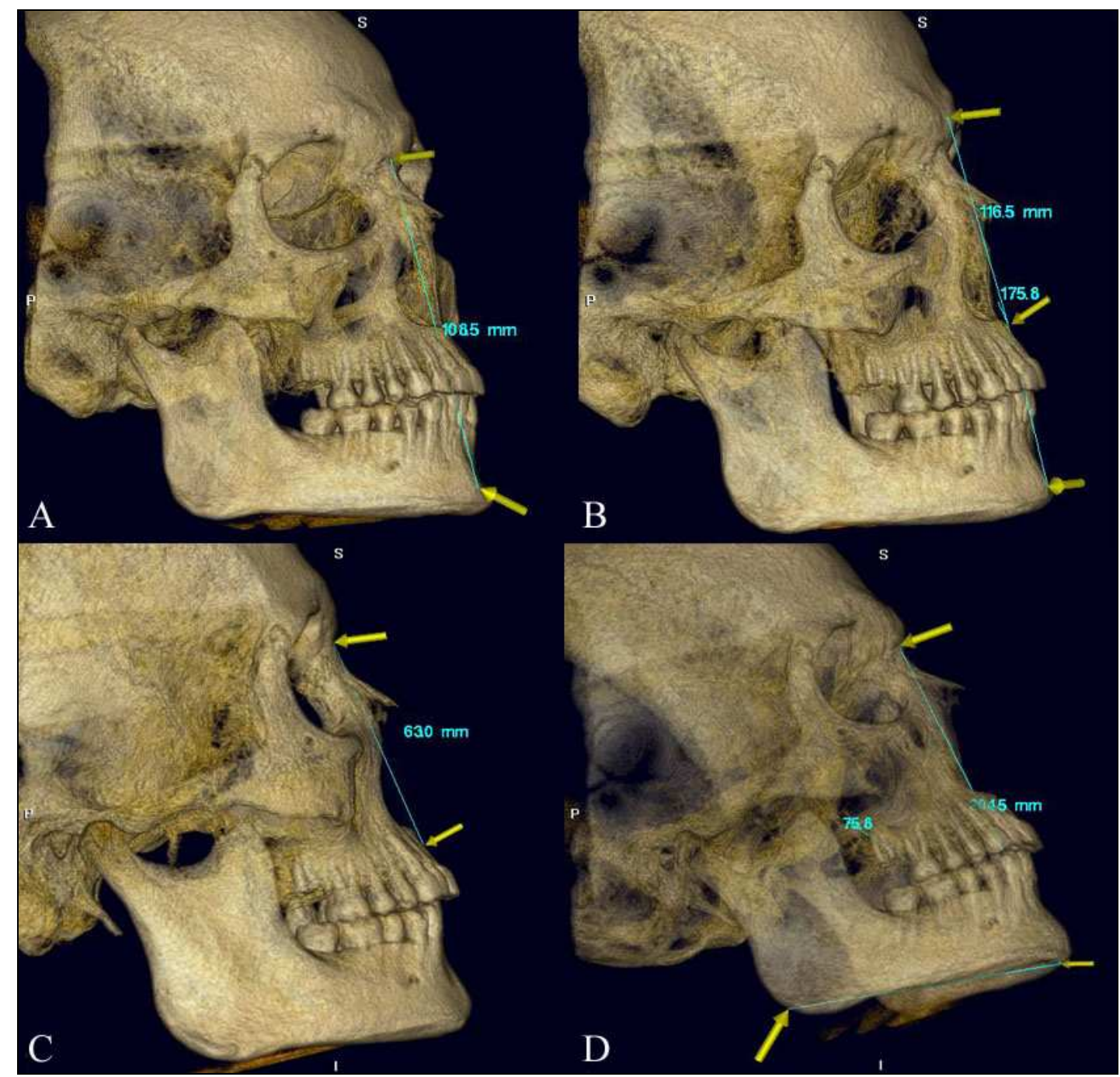

Figura 5 - Exemplos de medidas lineares e angulares obtidas nas imagens em 3D-TCFC: A e C, medidas lineares: $\mathrm{N}-\mathrm{Pg}=108,5 \mathrm{~mm}$ e $\mathrm{N}-\mathrm{A}=63,0 \mathrm{~mm}$. B e D, medidas angulares: G.ENA.Pg $=175,8^{\circ}$ e N.Me.Go $=75,8^{\circ}$, respectivamente

A análise das imagens requereu ajustes a partir de critérios pré-estabelecidos. Alguns parâmetros foram utilizados: 1. O lado direito foi usado para a marcação dos pontos, 2. Posições frontais e laterais foram padronizadas usando funções do programa e, 3. A mesma posição da cabeça foi utilizada para a obtenção das medidas (CAVALCANTI, ROCHA E VANNIER, 2004, LOPES, 2006; ACCORSI, 2007; LOPES et al., 2007, 2008). 


\subsection{ANÁLISE ESTATÍSTICA}

A análise dos dados consistiu na comparação entre as medidas físicas (padrão-ouro) com as obtidas na 3D-TCFC no programa Vitrea ${ }^{\circledR}$. Para facilitar a discussão, a precisão e a acurácia foram avaliadas segundo a mesma metodologia de trabalhos anteriores (LOPES et at., 2008). Erros sistemáticos das análises intra e interexaminador foram avaliados pelos testes t-pareado e Mann-Whitney, respectivamente. As medidas realizadas em 3D-TCFC foram confrontadas com as físicas através do teste de Mann-Whitney. A análise de variância (ANOVA) foi usada para a comparação entre os examinadores e as medidas físicas para cada medida separadamente. O nível de significância adotado foi de 5\%. 

5 RESULTADOS 



\section{RESULTADOS}

No total, 1260 medidas foram realizadas. Os resultados das medidas lineares e angulares serão apresentados separadamente.

\subsection{MEDIDAS LINEARES}

Os resultados das medidas lineares estão demonstrados nas Tabelas 3, 4 e 5. A Tabela 3 mostra a média, em milímetros, das medidas físicas e das em TCFC para ambos os examinadores.

Tabela 3 - Média, em milímetros, das medidas lineares no padrão-ouro (medidas físicas) e na 3D-TCFC para os dois examinadores

\begin{tabular}{cccc}
\hline \multirow{2}{*}{ Medidas } & Medidas Físicas & \multicolumn{2}{c}{ 3D-TCFC } \\
& (Padrão-ouro) & Examinador 1 & Examinador 2 \\
\cline { 3 - 4 } A-Pg & 56,1 & 55,7 & 56,8 \\
Co-A & 102,5 & 102,4 & 101,0 \\
Co-Pg & 123,9 & 122,4 & 120,6 \\
ENA-ENP & 49,5 & 50,1 & 49,4 \\
ENA-Me & 68,6 & 68,7 & 67,2 \\
ENA -N & 53,2 & 53,1 & 52,8 \\
ENA -A & 4,0 & 4,0 & 3,9 \\
N-A & 55,3 & 56,3 & 57,1 \\
N-B & 98,6 & 99,5 & 98,6 \\
N-Me & 119,2 & 119,7 & 118,3 \\
N-Pg & 111,0 & 111,8 & 112,8 \\
Po-Or & 79,5 & 78,6 & 81,4 \\
Zm-Zm & 93,6 & 93,8 & 91,2 \\
B-Me & 22,1 & 22,2 & 22,5 \\
Ba-N & 101,1 & 100,1 & 100,2 \\
\hline
\end{tabular}


Em média, as maiores diferenças absolutas e percentuais foram, respectivamente, $0,20 \mathrm{~mm}$ e $-0,15 \%$ (intra-examinador), e $0,31 \mathrm{~mm}$ e $0,19 \%$ (interexaminador) (Tabela 4). Para cada medida, em média, a maior diferença percentual foi $-3,43 \%$ (B-Me) para a análise intra-examinador e -3,61\% (Po-Or) para a interexaminador (Tabela 4).

Tabela 4 - Diferenças absolutas $(\mathrm{mm})$ e percentuais (\%) nas análises intra e interexaminador para cada medida linear

\begin{tabular}{|c|c|c|c|c|c|c|c|c|c|c|c|c|}
\hline & \multicolumn{4}{|c|}{$\begin{array}{c}\text { Precisão } \\
\text { intra-examinador } 1\end{array}$} & \multicolumn{4}{|c|}{$\begin{array}{c}\text { Precisão } \\
\text { intra-examinador } 2\end{array}$} & \multicolumn{4}{|c|}{$\begin{array}{c}\text { Precisão } \\
\text { interexaminador }\end{array}$} \\
\hline & \multicolumn{2}{|c|}{ Milímetro } & \multicolumn{2}{|c|}{$\%$} & \multicolumn{2}{|c|}{ Milímetro } & \multicolumn{2}{|c|}{$\%$} & \multicolumn{2}{|c|}{ Milímetro } & \multicolumn{2}{|l|}{$\%$} \\
\hline & Média & DP & Média & DP & Média & DP & Média & DP & Média & DP & Média & DP \\
\hline $\mathrm{A}-\mathrm{Pg}$ & $-0,48$ & 1,41 & $-0,89$ & 2,52 & $-0,61$ & 1,57 & $-1,18$ & 2,94 & $-1,11$ & 1,88 & $-2,01$ & 3,34 \\
\hline Co-A & 0,36 & 0,93 & 0,33 & 0,90 & 0,91 & 1,25 & 0,87 & 1,28 & 1,45 & 1,20 & 1,40 & 1,16 \\
\hline Co-Pg & $-0,24$ & 0,72 & $-0,20$ & 0,59 & 0,41 & 1,48 & 0,32 & 1,22 & 1,79 & 1,81 & 1,45 & 1,44 \\
\hline ENA-ENP & 0,11 & 0,57 & 0,20 & 1,08 & 0,21 & 0,96 & 0,43 & 1,97 & 0,75 & 1,05 & 1,47 & 2,07 \\
\hline ENA-Me & 0,00 & 0,59 & 0,00 & 0,86 & $-0,14$ & 1,16 & $-0,24$ & 1,73 & 1,48 & 1,39 & 2,18 & 2,02 \\
\hline ENA -N & 0,16 & 0,72 & 0,30 & 1,36 & $-0,21$ & 1,58 & $-0,40$ & 2,99 & 0,29 & 1,20 & 0,56 & 2,21 \\
\hline ENA -A & $-0,07$ & 0,37 & $-2,28$ & 9,70 & $-0,07$ & 0,33 & $-2,31$ & 7,91 & 0,06 & 0,40 & 0,70 & 9,45 \\
\hline $\mathrm{N}-\mathrm{A}$ & 0,38 & 1,06 & 0,64 & 1,87 & 0,59 & 2,04 & 0,90 & 3,76 & $-0,84$ & 2,24 & $-1,56$ & 4,13 \\
\hline $\mathrm{N}-\mathrm{B}$ & 0,81 & 2,26 & 0,76 & 2,23 & 2,54 & 1,75 & 2,56 & 1,75 & 0,93 & 2,70 & 0,92 & 2,69 \\
\hline $\mathrm{N}-\mathrm{Me}$ & 0,20 & 0,94 & 0,15 & 0,79 & $-0,09$ & 1,42 & $-0,07$ & 1,20 & 1,37 & 2,02 & 1,13 & 1,61 \\
\hline $\mathrm{N}-\mathrm{Pg}$ & 0,01 & 0,91 & $-0,01$ & 0,83 & $-0,47$ & 2,97 & $-0,37$ & 2,57 & $-0,99$ & 2,62 & $-0,97$ & 2,29 \\
\hline Po-Or & $-0,56$ & 1,05 & $-0,72$ & 1,32 & 2,17 & 4,98 & 2,54 & 6,25 & $-2,83$ & 3,18 & $-3,61$ & 4,11 \\
\hline $\mathrm{Zm}-\mathrm{Zm}$ & 0,07 & 1,19 & 0,13 & 1,31 & $-2,00$ & 2,89 & $-2,32$ & 3,28 & 2,63 & 2,99 & 2,75 & 3,19 \\
\hline B-Me & 0,19 & 0,57 & 0,71 & 2,66 & $-0,74$ & 1,45 & $-3,43$ & 6,49 & $-0,29$ & 1,36 & $-1,50$ & 6,36 \\
\hline Ba-N & 0,22 & 0,87 & 0,22 & 0,87 & 0,45 & 1,05 & 0,46 & 1,04 & $-0,02$ & 1,08 & $-0,02$ & 1,09 \\
\hline Total & 0,08 & 1,06 & $-0,04$ & 2,91 & 0,20 & 2,29 & $-0,15$ & 3,95 & 0,31 & 2,34 & 0,19 & 4,07 \\
\hline
\end{tabular}


A Tabela 5 apresenta a comparação entre as medidas lineares em 3D-TCFC e as medidas físicas para os dois examinadores. A diferença média entre as medidas físicas e em 3D variou entre 0,04 até -0,27 mm (Tabela 5).

Tabela 5 - Diferenças absolutas $(\mathrm{mm})$ e percentuais (\%) para a comparação entre as medidas lineares em 3D-TCFC e no padrão-ouro

\begin{tabular}{|c|c|c|c|c|c|c|c|c|}
\hline & \multicolumn{4}{|c|}{ Acurácia examinador 1} & \multicolumn{4}{|c|}{ Acurácia examinador 2} \\
\hline & \multicolumn{2}{|c|}{ Milímetro } & \multicolumn{2}{|c|}{$\%$} & \multicolumn{2}{|c|}{ Milímetro } & \multicolumn{2}{|c|}{$\%$} \\
\hline & Média & DP & Média & DP & Média & DP & Média & DP \\
\hline $\mathrm{A}-\mathrm{Pg}$ & $-0,36$ & 1,36 & -055 & 2,43 & 0,75 & 2,03 & 1,43 & 3,56 \\
\hline Co-A & $-0,06$ & 1,47 & $-0,06$ & 1,44 & $-1,50$ & 1,33 & $-1,47$ & 1,36 \\
\hline Co-Pg & $-1,45$ & 1,03 & $-1,15$ & 0,83 & $-3,25$ & 2,06 & $-2,58$ & 1,63 \\
\hline ENA-ENP & 0,68 & 1,16 & 1,38 & 2,30 & $-0,06$ & 1,17 & $-0,13$ & 2,41 \\
\hline ENA-Me & 0,16 & 0,93 & 0,26 & 1,28 & $-1,32$ & 1,25 & $-1,93$ & 1,86 \\
\hline ENA -N & $-0,07$ & 0,79 & $-0,10$ & 1,44 & $-0,37$ & 0,96 & $-0,68$ & 1,78 \\
\hline ENA -A & 0,00 & 0,40 & 0,09 & 10,52 & $-0,06$ & 0,29 & $-1,26$ & 7,13 \\
\hline $\mathrm{N}-\mathrm{A}$ & 0,93 & 0,93 & 1,72 & 1,72 & 1,77 & 2,33 & 3,31 & 4,52 \\
\hline N-B & 0,95 & 2,08 & 0,95 & 2,09 & 0,03 & 2,45 & 0,00 & 2,55 \\
\hline $\mathrm{N}-\mathrm{Me}$ & 0,45 & 1,01 & 0,38 & 0,84 & $-0,93$ & 1,66 & $-0,77$ & 1,33 \\
\hline $\mathrm{N}-\mathrm{Pg}$ & 0,85 & 1,73 & 0,78 & 1,52 & 1,84 & 2,73 & 1,76 & 2,44 \\
\hline Po-Or & $-0,87$ & 1,60 & $-1,09$ & 1,99 & 1,95 & 3,04 & 2,45 & 3,84 \\
\hline $\mathrm{Zm}-\mathrm{Zm}$ & 0,15 & 1,60 & 0,22 & 1,75 & $-2,47$ & 3,25 & $-2,54$ & 3,39 \\
\hline B-Me & 0,11 & 0,47 & 0,57 & 2,11 & 0,40 & 1,23 & 2,03 & 5,79 \\
\hline Ba-N & $-0,92$ & 1,19 & $-0,91$ & 1,17 & $-0,90$ & 1,07 & $-0,89$ & 1,05 \\
\hline Total & 0,04 & 1,41 & 0,17 & 3,19 & $-0,27$ & 2,41 & $-0,08$ & 3,77 \\
\hline
\end{tabular}

DP, desvio-padrão

Não houve diferenças estatisticamente significantes nas análises intra $(p=$ $0,281$ examinador $1, p=0,200$ examinador 2$)$ e interexaminador $(p=0,888)$ (Tabela 4). Também não foram observadas diferenças estatisticamente significantes na comparação entre TCFC e as medidas físicas para ambos os examinadores $(p=$ 0,968 and 0,915 , respectivamente) (Tabela 5). 


\subsection{MEDIDAS ANGULARES}

Os resultados das medidas angulares estão expressos nas Tabelas 6, 7 e 8. A Tabela 6 mostra a média em graus das medidas angulares do padrão-ouro (medidas físicas) e as em 3D-TCFC para ambos os examinadores.

Tabela 6 - Média, em graus, das medidas angulares no padrão-ouro (medidas físicas) e na 3D-TCFC para os dois examinadores

\begin{tabular}{cccc}
\hline Medidas & $\begin{array}{c}\text { Medidas Físicas } \\
\text { (Padrão-ouro) }\end{array}$ & Examinador 1 & Examinador 2 \\
\hline N.A.Pg & 167,4 & 167,4 & 166,8 \\
G.ENA.Pg & 167,7 & 168,1 & 169,2 \\
Co.Go.Me & 123,6 & 123,0 & 123,2 \\
N.Me.Go & 72,1 & 70,6 & 70,6 \\
N.A.B & 166,2 & 166,3 & 166,7 \\
N.ENA.Me & 160,1 & 159,0 & 152,9 \\
\hline
\end{tabular}

Em média, as maiores diferenças em graus e em porcentagem foram, respectivamente, $-0,36^{0}$ e $-0,33 \%$ (análise intra-examinador), e $-0,84^{0}$ e $-0,53 \%$ (análise interexaminador) (Tabela 7).

Tabela 7 - Diferenças absolutas $\left(^{0}\right)$ e percentuais (\%) nas análises intra e interexaminador para cada medida angular

\begin{tabular}{cccccccccccccc}
\hline & \multicolumn{3}{c}{$\begin{array}{c}\text { Precisão } \\
\text { intra-examinador 1 }\end{array}$} & \multicolumn{2}{c}{ intra-examinador 2 } & \multicolumn{3}{c}{ Precisão } & \multicolumn{2}{c}{$\begin{array}{c}\text { Precisão } \\
\text { interexaminador }\end{array}$} \\
\cline { 2 - 14 } & \multicolumn{2}{c}{ Grau } & \multicolumn{2}{c}{$\%$} & \multicolumn{2}{c}{ Grau } & \multicolumn{2}{c}{$\%$} & \multicolumn{2}{c}{ Grau } & \multicolumn{2}{c}{$\%$} \\
\cline { 2 - 14 } & Média & DP & Média & DP & Média & DP & Média & DP & Média & DP & Média & DP \\
\hline N.A.Pg & $-0,25$ & 0,94 & $-0,15$ & 0,57 & $-0,61$ & 1,90 & $-0,36$ & 1,14 & 0,57 & 2,09 & 0,33 & 1,25 \\
G.ENA.Pg & 0,38 & 0,87 & 0,23 & 0,51 & 0,25 & 1,78 & 0,15 & 1,04 & $-1,14$ & 2,27 & $-0,67$ & 1,33 \\
Co.Go.Me & 0,03 & 0,90 & 0,03 & 0,74 & $-0,36$ & 4,28 & $-0,38$ & 3,43 & $-0,18$ & 3,74 & $-0,19$ & 3,04 \\
N.Me.Go & 0,20 & 0,93 & 0,26 & 1,30 & $-0,61$ & 0,94 & $-0,89$ & 1,31 & 0,05 & 1,56 & 0,03 & 2,22 \\
N.A.B & 0,13 & 0,94 & 0,08 & 0,57 & $-0,69$ & 3,01 & $-0,44$ & 1,79 & $-0,39$ & 2,37 & $-0,23$ & 1,43 \\
N.ENA.Me & 0,17 & 1,05 & 0,11 & 0,65 & $-0,13$ & 1,34 & $-0,08$ & 0,83 & $-3,94$ & 1,60 & $-2,48$ & 0,99 \\
\hline Total & 0,11 & 0,94 & 0,09 & 0,76 & $-0,36$ & 2,43 & $-0,33$ & 1,79 & $-0,84$ & 2,75 & $-0,53$ & 2,02 \\
\hline
\end{tabular}


Para cada medida, a maior diferença em porcentagem foi -0,89\% (N.Me.Go) para a análise intra-examinador e $-2,48 \%$ (N.ENA.Me) para a interexaminador (Tabela 7).

A Tabela 8 apresenta a comparação entre as medidas angulares em 3DTCFC e as medidas físicas para os dois examinadores. A maior diferença entre a medida física e a 3D-TCFC para as medidas angulares foi $2,76^{\circ}$ (N.ENA.Me) e o valor mais baixo foi $-0,03 \%$ (N.A.Pg) (Tabela 8 ).

Tabela 8 - Diferenças absolutas $\left({ }^{0}\right)$ e percentuais (\%) na comparação entre as medidas angulares em 3D-TCFC e no padrão-ouro

\begin{tabular}{ccccccccc}
\hline & \multicolumn{3}{c}{ Acurácia examinador 1 } & \multicolumn{3}{c}{ Acurácia examinador 2 } \\
\cline { 2 - 10 } & \multicolumn{2}{c}{ Grau } & \multicolumn{2}{c}{$\%$} & \multicolumn{2}{c}{ Grau } & \multicolumn{2}{c}{$\%$} \\
\cline { 2 - 10 } & Média & DP & Média & DP & Média & DP & Média & DP \\
\hline N.A.Pg & $-0,05$ & 1,33 & $-0,03$ & 0,79 & $-0,62$ & 1,96 & $-0,37$ & 1,18 \\
G.ENA.Pg & 0,43 & 1,20 & 0,25 & 0,71 & 1,57 & 2,17 & 0,93 & 1,28 \\
Co.Go.Me & $-0,58$ & 2,05 & $-0,46$ & 1,64 & $-0,40$ & 3,84 & $-0,29$ & 3,06 \\
N.Me.Go & $-1,53$ & 0,94 & $-2,16$ & 1,36 & $-1,58$ & 1,18 & $-2,20$ & 1,64 \\
N.A.B & 0,08 & 1,39 & 0,04 & 0,84 & 0,46 & 3,33 & 0,27 & 2,01 \\
N.ENA.Me & $-1,18$ & 0,94 & $-0,74$ & 0,58 & 2,76 & 1,46 & 1,72 & 0,92 \\
\hline Total & $-0,47$ & 1,50 & $-0,52$ & 1,31 & 0,37 & 2,84 & 0,01 & 2,16 \\
\hline D.
\end{tabular}

$\mathrm{DP}$, desvio-padrão

Não houve diferenças estatisticamente significantes para as análises intra $(\mathrm{p}=$ $0,273$ examinador $1, p=0,165$ examinador 2$)$ e interexaminador $(p=0,603)$ (Tabela 7). Também não foram observadas diferenças estatisticamente significantes para a comparação entre a TCFC e as medidas físicas para ambos os examinadores $(\mathrm{p}=$ 0,844 e 0,700, respectivamente) (Tabela 8).

Diferenças relacionadas com as medidas separadamente também não foram estatisticamente significantes $(p>0,05)$. Os valores de $p$ variaram entre 0,137 (N.ENA.Me) a 0,978 (ENA-A) (Tabela 9). 
Tabela 9 - Valores de $p$ encontrados na comparação entre os dois examinadores e o padrão-ouro para cada medida separadamente (significância para $p<0,05$ )

\begin{tabular}{c|c}
\hline Medidas & Valores de $\boldsymbol{p}$ \\
\hline A-Pg & 0,921 \\
Co-A & 0,448 \\
Co-Pg & 0,286 \\
ENA-ENP & 0,686 \\
ENA-Me & 0,863 \\
ENA -N & 0,949 \\
ENA -A & 0,978 \\
N-A & 0,469 \\
N-B & 0,962 \\
N-Me & 0,823 \\
N-Pg & 0,597 \\
Po-Or & 0,244 \\
Zm-Zm & 0,357 \\
B-Me & 0,901 \\
Ba-N & 0,728 \\
N.A.Pg & 0,937 \\
G.ENA.Pg & 0,531 \\
Co.Go.Me & 0,910 \\
N.Me.Go & 0,443 \\
N.A.B & 0,961 \\
N.ENA.Me & 0,137 \\
\hline &
\end{tabular}


6 DISCUSSÃO 



\section{DISCUSSÃO}

A análise cefalométrica é realizada com base na marcação de pontos antropométricos para a obtenção de medidas lineares e angulares entre eles. É inquestionável como as imagens em 3D podem ser úteis no planejamento do tratamento (FARMAN e SCARFE, 2006; PINSKY et al., 2006; LAGRAVÈRE et al., 2008; SWENNEN e SCHUTYSER, 2006). Apesar disso, a exposição do paciente e a dose de radiação são fatores importantes a serem levados em consideração no momento da indicação da real necessidade de um exame radiográfico (LUDLOW e IVANOVIC, 2008).

Pontos craniométricos são pontos com localizações definidas em estruturas anatômicas e capazes de serem reproduzidos. O requisito mais importante para um ponto craniométrico é que ele seja facilmente identificado e localizado com grande acurácia e precisão. Oliveira et al. (2009) avaliaram a confiabilidade na identificação de pontos craniométricos em imagens de TCFC e afirmaram que a marcação tridimensional desses pontos em imagens de TCFC é reproduzível e consistente. Esta marcação é realizada através de programas de computação gráfica, onde as imagens em terceira dimensão são geradas. Programas de computação gráfica estão bem estabelecidos como um auxiliar importante na segmentação de imagens em 3D, na identificação e na marcação de pontos craniométricos para, assim, ocorrer a obtenção de medidas quantitativas (CAVALCANTI, ROCHA e VANNIER, 2004; LOPES, 2006; ACCORSI, 2007; LOPES et al., 2007, 2008).

Com o uso da TCFC na Odontologia, alguns estudos foram desenvolvidos para avaliar a acurácia de medidas lineares (PINSKY et al., 2006; LAGRAVÈRE et al., 2008; MOSHIRI et al., 2007; STRATEMANN et al., 2008; KUMAR et al., 2007), volumétricas (PINSKY et al., 2006) e angulares (LAGRAVÈRE et al., 2008; KUMAR et al., 2007) utilizando a TCFC.

Pinsky et al., em 2006, investigou a acurácia da TCFC em medidas lineares e volumétricas de defeitos ósseos e concluíram que a TCFC pode ser uma ferramenta de diagnóstico acurada para a avaliação de lesões ósseas pequenas. Os autores encontraram diferenças na acurácia variando de $-0,01$ a 0,27 $\mathrm{mm}$ para a largura e altura. Para o volume, identificaram um erro de até $2 \%$ do volume total dos defeitos. 
Esses achados estão de acordo com os de Periago et al. (2008), que estudou a acurácia de images de TCFC em medidas lineares e a considerou satisfatória clinicamente para estudos na região craniofacial. A maioria das diferenças foi menor que $2 \mathrm{~mm}$. A média do erro percentual para a 3D-TCFC foi 2,31\%. Houve diferença estatisticamente significante, mas os autores não a consideraram clinicamente relevante. Para as medidas lineares, em média, nossa maior diferença foi $0,31 \mathrm{~mm}$ $(0,19 \%)$ para todas as avaliações.

Stratemann et al. (2008) também encontraram alta acurácia em medidas lineares nas imagens de TCFC quando comparadas às medidas físicas (padrãoouro). O erro foi pequeno, variando de 0,00 a 0,07mm, e de 0,01 a 0,19\%. Eles consideraram esses erros mínimos e sem importância clínica. Nossos resultados em medidas lineares também foram considerados favoráveis, já que a média ficou entre 0,04 a 0,31 $\mathrm{mm}$ para precisão e acurácia.

Moshiri et al. (2007) verificou a acurácia de medidas lineares em TCFC e comparou com a radiografia cefalométrica. Eles não usaram imagens em 3D. As imagens em TCFC provaram ter maior acurácia que as radiografias cefalométricas quando comparadas ao padrão-ouro. Kumar et al. (2007) também confrontaram projeções em 2D oriundas da TCFC com radiografias cefalométricas convencionais. Eles fizeram medidas lineares e angulares e não encontraram diferenças estatisticamente significantes entre as duas modalidades. Porém, eles também não usaram imagens em 3D para realizar as medidas angulares, como em nosso estudo.

Outro trabalho testou a acurácia de medidas angulares e lineares em imagens em 3D-TCFC. Usando marcadores de titânio em uma mandíbula artificial, Lagravère et al. (2008) encontrou erros menores que $1 \mathrm{~mm}$ e 1 grau quando comparados ao padrão-ouro. Esses autores enfatizaram a possibilidade de análises quantitativas nesse tipo de imagem de alta qualidade. Em relação às medidas angulares, também encontramos diferenças médias menores que 1 grau $(-0,840$ ou $-0,53 \%)$ para todas as análises, comprovando a alta acurácia. Os dados desses autores, de uma forma geral, não diferiram dos nossos, mesmo eles não tendo usado pontos anatômicos verdadeiros. O fato de não terem que marcar os pontos craniométricos e apenas medirem as distâncias entre os marcadores eliminaria os erros inerentes à identificação do ponto e pode resultar em maior acurácia. 
O objetivo do nosso estudo foi avaliar medidas lineares e angulares realizadas diretamente em imagens de 3D-TCFC simulando a análise cefalométrica de rotina em situações clínicas reais. A diferença das pesquisas anteriores também consiste na identificação de pontos craniométricos usados em Ortodontia. Além disso, as medidas foram feitas sem o uso de esferas metálicas para guiar a identificação dos pontos craniométricos. A amostra foi de quinze crânios humanos secos e o exame em TCFC permitiu a aquisição da cabeça inteira, possibilitando uma análise craniofacial completa.

Estudos anteriores com a metodologia idêntica, apenas mudando a fonte - no caso, TC mutislice - encontraram diferenças variando de 2,05\% a 2,11\% para precisão e de $0,96 \%$ a $1,47 \%$ para acurácia em medidas lineares (LOPES et al., 2007). Nas medidas angulares, os valores variaram de 0,01 a $0,29 \%$ para precisão e de $-1,18 \%$ a $-0,89 \%$ para acurácia (LOPES et al., 2008). Esses resultados demonstram grande precisão e acurácia para as imagens em 3D-TC. Dessa forma, a acurácia de medidas lineares e angulares obtida pela TCFC é similar à TC multislice. A diferença média em milímetro/grau e em porcentagem para cada medida foi calculada no presente estudo. De uma forma geral, as diferenças foram pequenas e não foram estatisticamente significantes. Assim, parece que os nossos resultados refletem a realidade e as diferenças observadas não possuem significado clínico. Portanto, baseado na similaridade dos nossos resultados com os em TC multislice (LOPES et al., 2007 e 2008), podemos afirmar que a TCFC propicia imagens confiáveis para a análise cefalométrica.

Enquanto avanços tecnológicos têm melhorado a eficácia da radiação, simultaneamente há uma pressão para se obter imagens com alta resolução através de técnicas de escaneamento mais complexas. Esses dois fatores necessitam de doses de radiação maiores. Atenção especial tem sido dada na dose de radiação utilizada em técnicas tomográficas e seus efeitos biológicos.

A TCFC está se consolidando no campo da Ortodontia, representando um avanço tecnológico importante para o diagnóstico e tratamento (FARMAN e SCARFE, 2006; PERIAGO et al., 2008; SWENNEN e SCHUTYSER, 2006). Apesar disso, devemos nos atentar para as limitações dessa técnica no que diz respeito à qualidade da imagem, principalmente em 3D. O princípio de aquisição de dados em TCFC permite um volume parcial maior da região de interesse, já que a reconstrução 
não é simultânea à aquisição e as imagens em 3D ainda seguem a reconstrução pela técnica de superfície. Conseqüentemente, é preocupante a análise quantitativa para a aplicação na região craniofacial. Com o intuito de melhorar isso, aplicamos a reconstrução pela técnica de volume, similar ao método usado por Cavalcanti, Rocha e Vannier (2004) e por Lopes et al. (2007 e 2008), porém usamos a aquisição da imagem por TCFC. Nós observamos resultados similares de precisão e acurácia àqueles encontrados nesses trabalhos anteriores, provando também a importância de um pós-processamento de imagem 3D com qualidade para análise dos tecidos duros da região maxilofacial.

Pontos craniométricos em tecido mole não poderiam ser avaliados porque usamos um simulador de tecido mole (saco plástico com água) que não propiciava o contorno real da face. Yamashina et al. (2008) compararam a confiabilidade da TC multislice e da TCFC na mensuração do espaço aéreo da orofaringe. Os autores consideraram as imagens de TCFC acuradas para medir a superfície dos tecidos moles. Esse achado pode ser interessante para estudos posteriores in vivo envolvendo pontos craniométricos em tecidos moles comumente usados em análises faciais para fins ortodônticos.

Imagens reconstruídas em 3D a partir de TCFC permitiram o desenvolvimento de protocolos de medidas objetivas para o estudo do crânio e de suas alterações. Medidas lineares e angulares em 3D-TCFC servem de base para a avaliação objetiva de condições clínicas e permitem uma visão espacial que possibilita um melhor entendimento das alterações observadas nas imagens axiais. A aceitação dessas imagens para o estudo craniofacial está se difundindo. Além disso, refinamentos na técnica de TCFC, especialmente na qualidade da imagem, devem continuar ocorrendo. A previsão é que o uso da TCFC na região maxilofacial aumente, assim como a sua contribuição para o sucesso dos procedimentos em Odontologia. 
7 CONCLUSÃO 



\section{CONCLUSÃO}

A partir da análise dos resultados, podemos concluir que as medidas maxilofaciais lineares e angulares obtidas por TCFC foram consideradas precisas e a acuradas. Desta forma, a TCFC pode ser utilizada na execução de medidas craniométricas em imagens tridimensionais. 

REFER $\hat{E N C I A S}$ 



\section{REFERÊNCIAS}

Accorsi MAO. Comparação de grandezas cefalométricas obtidas por meio de telerradiografias e tomografias computadorizadas multislice em crânios secos humanos [Dissertação de Mestrado]. São Paulo: Faculdade de Odontologia da USP; 2007.

Cavalcanti MGP, Vannier MW. Quantitative analysis of spiral computed tomography for craniofacial clinical applications. Dentomaxillofac Radiol 1998; 27:344-350.

Cavalcanti MG, Ruprecht A, Vannier MW. 3D volume rendering using multislice CT for dental implants. Dentomaxillofac Radiol 2002;31:218-23.

Cavalcanti MGP, Rocha SS, Vannier MW. Craniofacial measurements based on 3DCT volume rendering. Implications for clinical applications. Dentomaxillofac Radiol 2004; 33:170-176.

Farman AG. Scarfe WC. Development of imaging selection criteria and procedures should precede cephalometric assessment with cone-beam computed tomography Am J Orthod Dentofacial Orthop 2006; 130:257-265.

Jamison PL, Ward RE. Brief communication: measurement size, precision, and reability in craniofacial anthropometry: bigger is better. Am J Phys Anthropol 1993; 90:495-500.

Kragskov J, Bosch C, Gyldensted C, Sindet-Pedersen S. Comparison of the reliability of craniofacial anatomic landmarks based on cephalometric radiographs and threedimensional CT scans. Cleft Palate Craniofac J 1997; 34:111-116.

Kumar V, Ludlow JB, Mol A, Cevidanes L. Comparison of conventional and cone beam CT synthesized cephalograms. Dentomaxillofac Radiol 2007; 36:263-9.

Lagravère MO, Carey J, Toogood RW, Major PW. Three-dimensional accuracy of measurements made with software on cone-beam computed tomography images. Am J Orthod Dentofacial Orthop 2008; 134:112-6.

Lopes PML. Validação de medidas ósseas craniofaciais por meio da tomografia computadorizada multislice em 3D [Tese de Doutorado]. São Paulo: Faculdade de Odontologia da USP; 2006. 
Lopes PML, Perrella A, Moreira CR, Rino Neto J, Cavalcanti MG. Aplicação de medidas cefalométricas em 3D-TC. Rev Dent Press Ortodon Ortop Facial 2007. Jul./ago;12(4):99-106.

Lopes PM, Moreira CR, Perrella A, Antunes JL, Cavalcanti MG. 3D volume rendering maxillofacial analysis of angular measurements by multislice CT. Oral Surg Oral Med Oral Pathol Oral Radiol Endod 2008; 105:224-30.

Ludlow JB, Ivanovic M. Comparative dosimetry of dental CBCT devices and 64-slice $\mathrm{CT}$ for oral and maxillofacial radiology. Oral Surg Oral Med Oral Pathol Oral Radiol Endod 2008; 106:106-14.

Moshiri M, Scarfe WC, Hilgers ML, Scheetz JP, Silveira AM, Farman AG. Accuracy of linear measurements from imaging plate and lateral cephalometric images derived from cone-beam computed tomography. Am J Orthod Dentofacial Orthop 2007; 132:550-60.

Mozzo P, Procacci C, Tacconi A, Martini PT, Andreis IA. A new volumetric CT machine for dental imaging based on the cone-beam technique: preliminary results. Eur Radiol 1998; 8:1558-64.

Naitoh M, Katsumata A, Nohara E, Ohsaki C, Ariji E. Measurement accuracy of reconstructed 2-D images obtained by multi-slice helical computed tomography. Clin Oral Implants Res 2004;15:570-4.

Oliveira AE, Cevidanes LH, Phillips C, Motta A, Burke B, Tyndall D. Observer reliability of three-dimensional cephalometric landmark identification on cone-beam computerized tomography. Oral Surg Oral Med Oral Pathol Oral Radiol Endod. 2009 Feb;107(2):256-65.

Periago DR, Scarfe WC, Moshiri M, Scheetz JP, Silveira AM, Farman AG. Linear accuracy and reliability of cone beam CT derived 3-dimensional images constructed using an orthodontic volumetric rendering program. Angle Orthod 2008; 78:387-95.

Pinsky HM, Dyda S, Pinsky RW, Misch KA, Sarment DP. Accuracy of threedimensional measurements using cone-beam CT. Dentomaxillofac Radiol 2006; 35:410-6.

Scarfe WC, Farman AG, Sukovic P. Clinical applications of cone-beam computed tomography in dental practice. J Can Dent Assoc 2006 Feb;72:75-80. 
Stratemann SA, Huang JC, Maki K, Miller AJ, Hatcher DC. Comparison of cone beam computed tomography imaging with physical measures. Dentomaxillofac Radiol 2008; 37:80-93.

Sukovic $\mathrm{P}$. Cone beam computed tomography in craniofacial imaging. Orthod Craniofac Res 2003; 6 Suppl 1:31-6.

Swennen GR, Schutyser F. Three-dimensional cephalometry: Spiral multi-slice vs cone-beam computed tomography. Am J Orthod Dentofacial Orthop 2006; 130:410416.

Yamashina A, Tanimoto $\mathrm{K}$, Sutthiprapaporn $\mathrm{P}$, Hayakawa $\mathrm{Y}$. The reliability of computed tomography (CT) values and dimensional measurements of the oropharyngeal region using cone beam CT: comparison with multidetector CT. Dentomaxillofac Radiol. 2008;37:245-51. 

ANEXO 

ANEXO A - Parecer de aprovação do Comitê de Ética em Pesquisa da Faculdade de Odontologia da Universidade de São Paulo.

\section{UNIVERSIDADE DE SÃO PAULO FACULDADE DE ODONTOLOGIA}

\section{PARECER DE APROVAÇÃO \\ Protocolo 133/07}

O grupo de trabalho indicado pelo Comitê de Ética em Pesquisa APROVOU o protocolo de pesquisa "VALIDAÇÃO DE MEDIDAS MAXILOFACIAIS POR MEIO DA TOMOGRAFIA COMPUTADORIZADA POR FEIXE CÔNICO EM 3D UTILIZANDO PROGRAMAS DE COMPUTAÇÃO GRÁFICA", de responsabilidade da Pesquisadora CARLA RUFFEIL MOREIRA, sob orientação do Professor Doutor MARCELO DE GUSMÃO PARAíSO CAVALCANTI.

Tendo em vista a legislação vigente, devem ser encaminhados a este Comitê relatórios anuais referentes ao andamento da pesquisa e ao término cópia do trabalho em "cd". Qualquer emenda do projeto original deve ser apresentada a este CEP para apreciação, de forma clara e sucinta, identificando a parte do protocolo a ser modificada e suas justificativas.

São Paulo, 04 de outubro de 2007

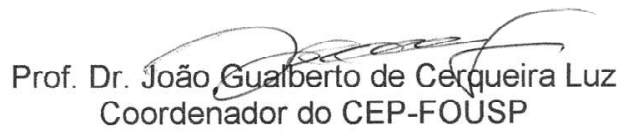

Av. Prof. Lineu Prestes, 2227 - Cidade Universitária "Armando de Salles Oliveira" CEP 05508-900 São Paulo - SP - Diretoria Telefax: (011) 3094- 0062/3091-7817/3091-7860 - Compras (011) 3091-7895 Impresso no S. D.O 